\title{
Heart failure in cancer: role of checkpoint inhibitors
}

\author{
Murilo Delgobo ${ }^{1,2}$, Stefan Frantz ${ }^{1,2}$ \\ ${ }^{1}$ Department of Internal Medicine I, University Hospital Würzburg, Würzburg, Germany; ${ }^{2}$ Comprehensive Heart Failure Center, University of \\ Würzburg, Würzburg, Germany \\ Contributions: (I) Conception and design: All authors; (II) Administrative support: None; (III) Provision of study materials or patients: None; \\ (IV) Collection and assembly of data: All authors; (V) Data analysis and interpretation: All authors; (VI) Manuscript writing: All authors; (VII) Final \\ approval of manuscript: All authors. \\ Correspondence to: Stefan Frantz, MD. Department of Internal Medicine I, University Hospital Würzburg Oberdürrbacher Straße 6, 97080 \\ Würzburg, Germany. Email: frantz_s@ukw.de.
}

\begin{abstract}
The introduction of immune checkpoint inhibitors have greatly improved clinical outcomes in several cancer types, revolutionizing the management of a wide variety of tumors endowed with poor prognosis. Despite its success, high grade immune related adverse events were observed in patients treated with checkpoint inhibitors. While cardiotoxicity was largely underestimated in initial studies, numerous reports of fulminant myocarditis and fatal heart failure $(\mathrm{HF})$ have been recently described. In this review we discuss possible mechanisms involved in cardiac toxicity triggered by inhibition of cytotoxic $\mathrm{T}$ lymphocyte antigen 4 (CTLA-4) and programmed cell death 1 (PD-1) pathway, the most prominent checkpoint inhibitors available in the clinic. Major cardiovascular events associated with checkpoint inhibitors adds another layer of complexity in cancer therapy and urges for an interdisciplinary approach between oncologists, cardiologists, and immunologist.
\end{abstract}

Keywords: Myocarditis; cardiac toxicity; checkpoint inhibitors; cardio-oncology

Submitted Sep 20, 2018. Accepted for publication Oct 02, 2018.

doi: $10.21037 /$ jtd.2018.10.07

View this article at: http://dx.doi.org/10.21037/jtd.2018.10.07

\section{Introduction}

The interaction of the heart with cancer and cancer treatment has recently become a topic of high interest in scientific community. Indeed recent findings suggest that heart failure (HF) can trigger tumor growth: The group of R. de Boer demonstrated that presence of HF enhanced tumor growth (1). On the other side, there are several anti-cancer medications that might lead to HF. Therefore, the discipline Cardio-oncology was coined to describe strategies on detection, monitoring, and treatment of cardiovascular diseases occurring as a side effect of chemotherapy and radiotherapy (2). Cardiotoxicity can occur as a result of cancer therapy or be accelerated in patients which present traditional risk factors. Earlier studies showed how cancer patients exposed to anthracyclines developed HF and further studies on trastuzumab (anti-HER-2) expanded the notion of cardiotoxicity triggered by chemotherapy $(3,4)$.
Consequently, the European Society of Cardiology published two position papers on the management of cardiotoxicity that develops during cancer in the past two years $(5,6)$. While those reports provide strategies for cardiotoxicity triggered by anthracyclines, antimetabolites, anti-HER2, anti-VEGF antibodies and tyrosine kinase inhibitors, a gap still exists regarding the so-called immune checkpoint inhibitors.

The emergence of immune target therapies in oncology since the early 1990s [interferons (IFNs) and IL-2 therapy] has revolutionized the management of advanced-stage malignancies of various histological types with previously poor prognosis. During cancer progression, tumor cells accumulate mutations which can result in abnormal or neoantigen expression. Those antigens are readily recognized by innate and adaptive immune cells, leading to killing of tumor cells and protective immunity. However, tumor cells can avoid or subvert host immune response by exploiting a large variety of immune-escape mechanisms, including changes 


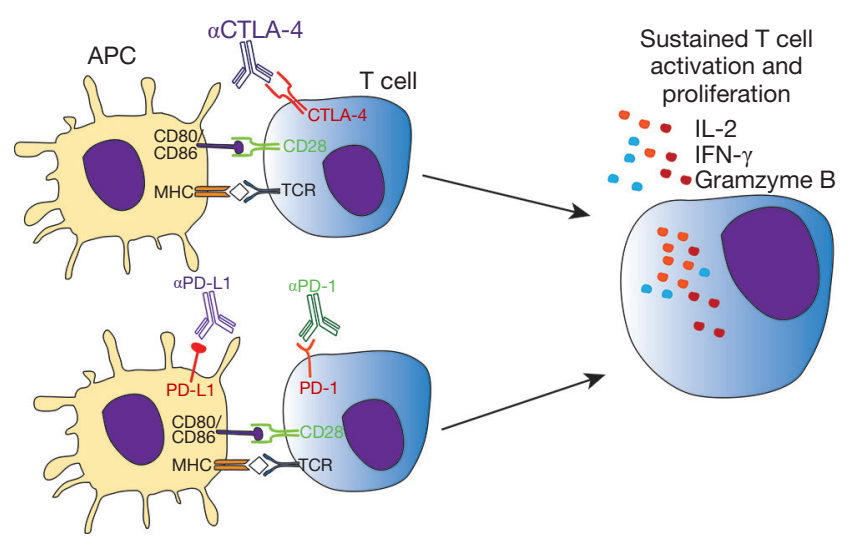

Figure 1 Sustained activation of $\mathrm{T}$ cells by immune checkpoint inhibitors. T cell activation by APCs occurs through 2 distinct signals: (I) antigen sequences within MHC molecule are recognized by TCR; (II) co-stimulatory molecules expressed by APCs binds CD28 receptor on $\mathrm{T}$ cells, strengthening TCR signaling and promoting $\mathrm{T}$ cell activation and survival. Continuous antigen presentation leads to upregulation of CTLA-4 and PD-1 in T cells, which promotes cell suppression, regulatory phenotype or anergy/ apoptosis. As a result, antibody mediated inhibition of CTLA-4, PD-1, or its ligand (PD-L1) extends T cell activation, survival, and proliferation in the context of antigen presentation. APC, antigenpresenting cell; MHC, major histocompatibility complex; TCR, T-cell receptor; CTAL-4, cytotoxic T lymphocyte antigen 4.

in the expression of MHC, NK ligands, FAS receptor, and immune-checkpoint molecules (7). While those pathways are necessary for peripheral tolerance maintenance, in cancer context they promote tumor growth and ultimately host death $(7,8)$. In particular, seminal studies conducted by Allison and Chen group demonstrated the ability of tumor cells to hijack immune tolerance through cytotoxic T lymphocyte-associated antigen 4 (CTLA-4) and programmed cell death 1 (PD-1) molecules respectively, which profoundly dampen the antitumor functions of $\mathrm{T}$ cells $(9,10)$. In this context, neutralization of CTLA-4 results in tumor rejection, including pre-established tumors, promoting protective immunity against secondary exposure to cancer cells (9). Remarkably, clinical usage of neutralizing monoclonal antibodies targeting those immune checkpoint molecules as Ipilimumab (anti-CTLA-4), nivolumab (anti-PD-1), and atezolizumab (anti-PD-L1) have largely improved the prognosis of solid and hematological tumors, such as melanoma and multiple myeloma, cancer types endowed with poor prognosis (11) (Figure 1 and Table 1).

Although immune checkpoint inhibitors (ICI) treatment brought an unprecedented advance in cancer therapy, a wide variety of immune related adverse effects in different organ systems were reported in patients undergoing therapy (12). Of notice, several cases of autoimmune myocarditis and fatal HF were reported in cancer patients treated with ICI during the last years (13). While cardiotoxicity (hypertension, myocarditis, thromboembolism, arrhythmias and fibrosis) induced by conventional anti-neoplastic drugs (e.g., anthracyclines, anti-metabolites, Her-2 inhibitors, anti-VEGF) have been historically reported [reviewed in (14)], new evidence points at cardiac adverse effects of biological drugs targeting specific signaling pathways.

\section{CTLA4}

Activation of antigen specific $\mathrm{T}$ lymphocytes are a hallmark of adaptive immune response. Briefly, naïve T cells require two distinct signals in order to proliferate and differentiate to effector cells. The first signal comes from the interaction of TCR with its cognate antigen, presented in class I or II MHC context. The second signal is conferred by co-stimulatory molecules expressed in antigen-presenting cells (APCs), known as B7 molecules (CD80 and CD86). In $\mathrm{T}$ cells, CD28 recognizes $\mathrm{B} 7$ molecules, promoting along with the first signal, $\mathrm{T}$ cell activation. Besides CD28, CTLA-4 also recognizes B7 molecules but negatively impacts $\mathrm{T}$ cell activation, leading to cell arrest, regulatory phenotype or apoptosis (15). CTLA-4 is normally confined to vesicles in naïve $T$ cells, homing to extracellular membrane following $\mathrm{T}$ cell activation. Importantly, both CD28 and CTLA-4 competes for B7 binding, where CTLA-4 has significantly higher affinity than its counterpart. For this reason the tight spatial and temporal regulation of CTLA-4 expression is critical for determining the outcome of $\mathrm{T}$ cell response (16). Those mechanisms are essential for mounting a protective immune response while controlling inflammation, tissue damage, and promoting immune tolerance (Figure 2). This becomes evident in the study of Tivol and coworkers, where mice with knockout for CTLA4 develop massive polyclonal expansion of peripheral $\mathrm{T}$ cells and fatal multiorgan tissue destruction (17). In addition, treatment of mice with anti-CD4 antibody abolishes lymphoproliferation and autoimmunity, suggesting that $\mathrm{CD} 4^{+} \mathrm{T}$ cells initiate the phenotype in CTLA-4 KO mice (18). Work of Sakaguchi highlights the role of CTLA-4 in controlling Treg homeostasis. Specific 
Table 1 Different classes of Immune checkpoint inhibitors

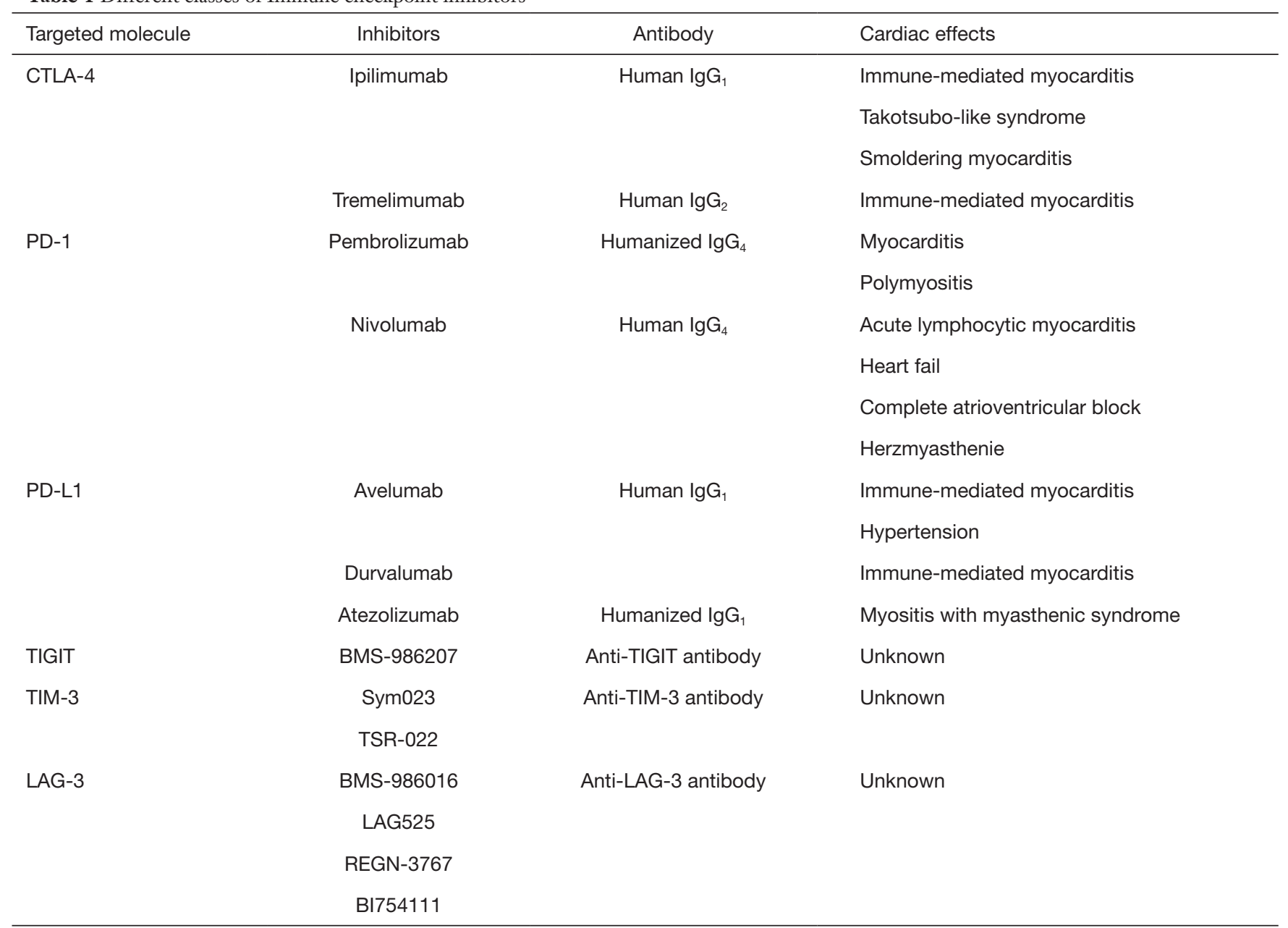

CTAL-4, cytotoxic T lymphocyte antigen 4; TIGIT, T cell immunoreceptor Ig and ITIM domains; TIM-3, T-cell immunoglobulin and containing-3; LAG-3, lymphocyte-activation gene 3; PD-1, programmed cell death protein 1; PD-L1, programmed death-ligand 1.

deletion of CTLA-4 in $\mathrm{FoxP}^{+}$cells results in spontaneous development of systemic lymphoproliferation and fatal $\mathrm{T}$ cell mediated autoimmune disease (19). In humans, CTLA-4 signaling also controls $\mathrm{T}$ cell homeostasis, as demonstrated by Treg dysregulation, hyperactivation of effector T cells, and lymphocytic infiltration in brain, lung, and gut tissue of patients carrying Ctla4 mutation (20).

Whereas immune checkpoint molecules are essential for maintaining immune tolerance and preventing autoimmunity, strong $\mathrm{T}$ cell suppression can be unfavorable for the host. Pre-clinical and clinical data have shown that $\mathrm{T}$ cell functions are impaired in several types of cancers, where those cells display an unresponsiveness to tumor antigens (anergy/ exhaustion) and a regulatory phenotype (21). Co-stimulatory molecules, which are expressed by APCs, can also be found in different types of tumor cells and further activate immune checkpoint receptors $(22,23)$. As a strategy to increase $\mathrm{T}$ cell response towards tumor cells and enhance antitumor immunity, pioneering work conducted by Allison showed that inhibition of CTLA-4 signaling promotes tumor rejection. Blocking of CTLA-4 increased T effector cells activity, promoted Treg depletion and mediated Fcreceptor dependent tumor rejection (9). In 2010, a phase 3 study reported an improved overall survival in patients with metastatic melanoma treated with Ipilimumab (anti-CTLA-4), imposing a new paradigm for cancer immunotherapy (24). In the same year, Ipilimumab was approved by the Food and Drug Administration and in 2011 by the European Medicines Agency to treat melanoma.

Although ICI treatment has led to ground breaking 


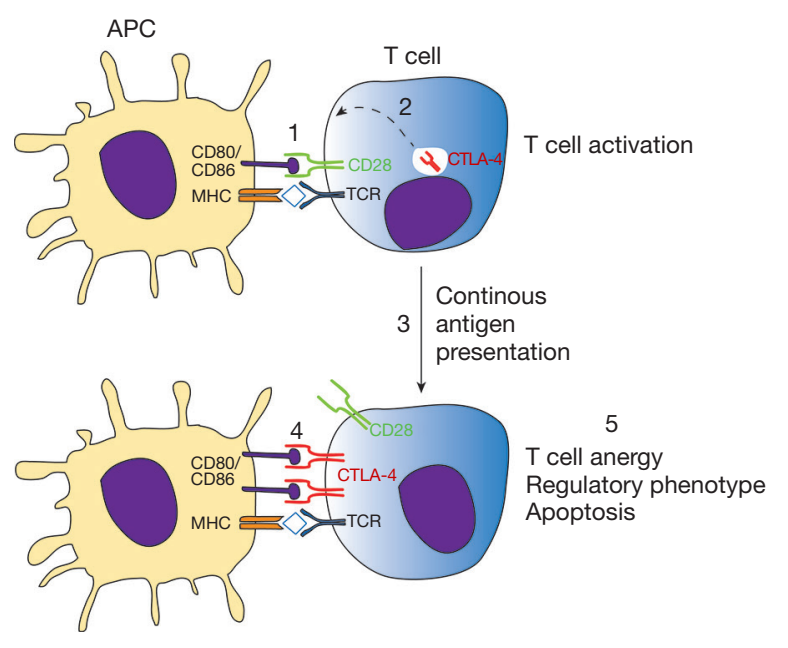

Figure 2 Suppression of T cell response by CTLA-4 signaling. CTLA-4 is restricted within vesicles in naïve $\mathrm{CD}^{+} \mathrm{T}$ cells. Following T cell activation by TCR and CD28 molecules [1], CTLA-4 is recruited to T cells' membrane [2]. In a context of continuous antigen presentation [3], CTLA-4 binds with higher affinity to CD80/CD86 molecules [4], resulting in suppression of $\mathrm{T}$ cell functions, regulatory phenotype, or apoptosis. TCR, T-cell receptor; CTAL-4, cytotoxic T lymphocyte antigen 4.

improvements in cancer therapy, several immune related adverse events (IRAE) were reported. Due to their critical role in immune tolerance maintenance, therapeutic blockade of checkpoint molecules can give rise to inflammatory or autoimmune side effects. In its first report, $10 \%$ to $15 \%$ of patients experienced grade 3 and 4 IRAE; 14 deaths (2.1\%) were related to Ipilimumab treatment (24). Whereas not described in phase 3 study, fulminant myocarditis after Ipilimumab and other ICIs treatment has recently caught attention from physicians and scientific community (25). Since then, a growing body of evidence has emerged over the last years illustrating cardiac toxicity of ICI, with 101 cases of severe myocarditis reported in 2017 (13).

A link between CTLA-4 function and cardiac homeostasis has long been observed in mouse models which do not express this molecule and succumb to death due to myocarditis and pancreatitis (17). In transplant context, CTLA-4 blockade accelerates the acute rejection of cardiac allografts, in a mechanism independent of CD28 signaling. Increased lymphocyte infiltration followed by upregulation of IL-6 and IFN- $\gamma$ transcripts on allografts suggests autoreactive $\mathrm{T}$ cell expansion, augmented by CTLA-4 blocking (26). On the other hand, CTLA-4 based conditioning resulted in cardiac allograft tolerance, as demonstrated by the absence of obliterative arteriopathy and lymphocyte hyporesponsiveness in graft tolerant animals (27). In a mouse model of coxsackievirus B3 (CVB3) induced myocarditis, Frisancho-Kiss and coworkers demonstrated that CTLA-4 controls regulatory $\mathrm{T}$ cell population and heart inflammation in a Tim-3 dependent mechanism (28). Corroborating those results, Han and coworkers found reduced IFN- $\gamma$ levels and increased survival in CVB3 infected mice treated with CTLA-4 agonist (29). Moreover, the Lichtman group showed that CTLA-4 regulates $\mathrm{CD} 8^{+} \mathrm{T}$ cell responses in myocarditis, where CTLA-4 ablation combined with IL-12 driven differentiation synergistically increased cardiac pathogenicity of $\mathrm{CD}^{+} \mathrm{T}$ cells (30).

Evidence in humans also depicts the pivotal role of $\mathrm{T}$ cells in cardiac diseases. In a study conducted by the German Heart Failure Network, the G/G genotype of Thr17Ala CTLA-4 variant was significantly more frequent in patients with dilated cardiomyopathy $(14.7 \%)$ versus $7 \%$ in control group (31). The G/G genotype was also more frequent in patients that developed Rheumatic Heart Disease as a sequela of Acute Rheumatic Fever, illustrating that uncontrolled $\mathrm{T}$ cell activation can promote cardiac tissue damage (32). In contrast, genotypes and haplotypes reported to increase CTLA-4 expression were associated with asymptomatic form of Chagas disease, whereas genotypes associated with reduced function were more present in patients with a mixed form of disease (cardiodigestive form) (33). Taken together, those observations highlight the importance of $\mathrm{T}$ cell regulation by immune checkpoint molecules in cardiac homeostasis (Figure 3).

The importance of heart-T cell axis was further addressed in patients receiving neutralizing antibodies against CTLA-4. In 2016 two cases of fatal myocarditis were reported in patients receiving Ipilimumab and nivolumab (anti-PD-1). Myositis with rhabdomyolysis followed by massive macrophage and $\mathrm{T}$ cell infiltration were found in both patients. $\mathrm{T}$ cell clones infiltrating the myocardium were identical to those in tumors and composed of both $\mathrm{CD}^{+}$and $\mathrm{CD} 8^{+}$cells, suggesting unspecific $\mathrm{T}$ cell autoimmunity mediating myocarditis (34). Takotsubo-like syndrome was also reported in a patient with metastatic melanoma after receiving four doses of Ipilimumab, suggesting that autoimmune myocarditis triggered by antiCTLA-4 treatment could be a cause of apical ballooning and cardiomyopathy (35). The same dose regimen was also found to induce acute myocarditis in patient with malignant 


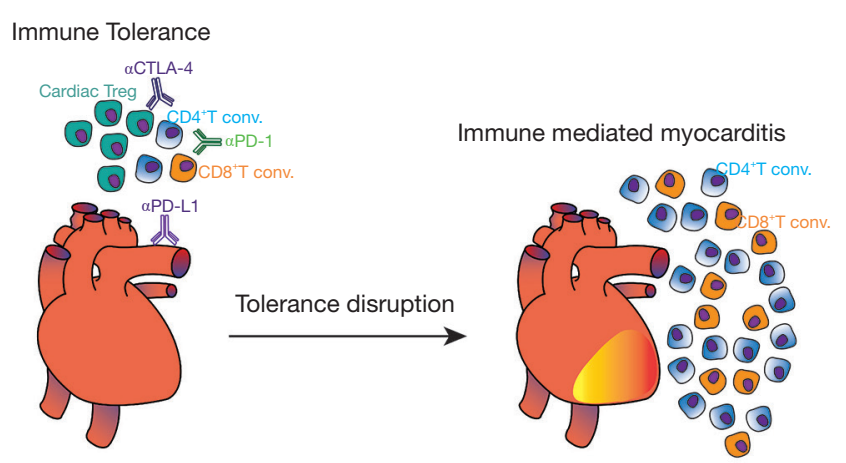

Figure 3 Autoimmune myocarditis triggered by Immune checkpoint inhibitors. Cardiac regulatory $\mathrm{T}$ cells, among $\mathrm{CD} 4^{+}$ and $\mathrm{CD}^{+} \mathrm{T}$ cells are present in the heart and draining lymph nodes under physiological condition, exerting protective immune response. Blockade of checkpoint molecules CTLA-4/PD-1 in $\mathrm{T}$ cells and PD-L1, expressed on endothelial and cardiac muscle cells, disrupts tissue tolerance, inhibits Treg functions and initiates conventional $\mathrm{T}$ cell activation and proliferation. Uncontrolled $\mathrm{T}$ cell response in the heart promotes inflammation and tissue damage. CTAL-4, cytotoxic T lymphocyte antigen 4.

melanoma, which lead to biventricular failure (36). In another study, post mortem analysis of heart tissue demonstrated findings consistent with giant cell myocarditis, from a patient that presented with steroidrefractory myocarditis after Ipilimumab treatment. While high clonality was found in metastatic lung tissue, low TCR repertoire was found in the heart $(37,38)$. In a comprehensive analysis of ICI associated myocarditis, 35 patients were identified from 2013 until July 2017, corresponding to $1.14 \%$ of patients receiving ICI. Importantly, myocarditis onset was in median 34 days after starting ICI and following 102 days, $46 \%$ of those patients developed major adverse cardiac events. Their findings portrait myocarditis after ICI therapy as an early event, with malignant course, which responds to high doses of steroids. In comparison to controls, myocarditis cases had a higher prevalence of diabetes mellitus and higher body mass index (39). Late onset of cardiac adverse effects was also reported in metastatic melanoma patient after twelve weeks of last ipilimumab cycle administration. ICI treatment was accompanied by acute fibrinous pericarditis with mixed inflammatory infiltrates in pericardial wall and surface fibrin deposition (40). Norwood and coworkers reported a case of smoldering myocarditis soon after (17 days) of ipilimumab plus nivolumab treatment. Besides no cardiac clinical symptoms were present, troponin I levels were 13 times the upper limit and endomyocardial biopsy revealed fibrosis with lymphocytic inflammation, predominantly comprised of $\mathrm{CD}^{+} \mathrm{T}$ cells (41). In summary, inflammatory response in the heart following CTLA-4 inhibition can occur quickly after treatment and progress to life-threatening condition.

\section{PD-1 and PD-L1}

Programmed cell death 1 receptor and its cognate ligands, along with CTLA-4, constitutes the major group of immune checkpoint molecules which counteract $\mathrm{T}$ cell activation program. Similarly to CTLA-4, PD-1 is not normally expressed on naïve T cells, whereas TCR engagement induces its expression. PD-1 negatively regulates TCR and CD28 signaling pathways when bound to its ligands PD-L1 (B7-H1) and/or PD-L2 (B7-DC) (42). In the absence of antigen clearance, PD-1 expression is sustained, leading to T cell exhaustion, a state of unresponsiveness towards the cognate antigen and/or apoptosis (43). While CTLA-4 expression is more restricted to T cells, PD-1 is also present on B cells, $\mathrm{NK}$ cells and in some myeloid cells. $\mathrm{CD} 8^{+} \mathrm{PD}-1^{+}$cells are found in the circulation of healthy human subjects (42). In that way, PD-1 signaling can promote different responses depending on cell type and microenvironment involved. In particular, several tumor cells express PD-L1, which may dampen antitumor $\mathrm{T}$ cell response. Early works of Minato group showed indeed that PD-L1 expression in P815 tumor cells rendered them less susceptible to $\mathrm{T}$ cell antigen receptor-mediated lysis. Moreover, growth of myeloma cell lines in syngeneic mice was significantly inhibited by administration of neutralizing PD-L1 antibody (23). Involvement of PD-1 in tumor immune escape was also reported in humans, where $\mathrm{CD} 8^{+} \mathrm{T}$ cells from melanoma patients present increased expression of PD-1 and impaired cytotoxicity against tumor cells (44). Regarding its ability to inhibit $T$ cell activation against tumor cells, neutralizing antibodies against PD-1 (nivolumab) and their respective ligand PD-L1 (atezolizumab) were developed and tested in tumors with poor prognosis. Notably, $20 \%$ to $25 \%$ of patients presenting either melanoma, non-small cell lung, or renal cancer obtained objective responses, lasting more than one year. Nivolumab also increased the survival of patients with previously untreated melanoma, with 5.1 months of progression-free survival against 2.2 months in dacarbazine group $(45,46)$. It was therefore approved in December 2014 for the treatment of unresectable or metastatic melanoma by FDA. 
Despite its success in clinical trials, similarly to antiCTLA-4 treatment, PD-1 blocking lead to $14 \%$ of grade 3 and 4 IRAE, where in $1 \%$ of patients drug related adverse effects led to patient death $(45,46)$. Although cardiovascular adverse effects were also unnoticed in initial studies with anti-PD-1 therapy in humans, recent reports have demonstrated a link between PD-1 treatment and autoimmune myocarditis, especially with anti-CTLA-4 treatment (47). The role of PD-1 controlling autoimmunity was illustrated in a mouse knockout model, which developed spontaneous lupuslike proliferative arthritis and glomerulonephritis (48). In NOD (nonobese diabetic) mice, PD-1 deletion accelerates the onset and frequency of type I diabetes, with strong Th1 polarization and $\mathrm{T}$ cell accumulation into islets (49). Strikingly, deletion of PD-1 in BALB/C mice resulted in dilated cardiomyopathy with severely impaired contraction and sudden death by congestive heart failure. The lymphocyte compartment was necessary for pathology induction, where PD-1 and RAG2 double knockout did not developed the disease (50). Furthermore, high titer of autoantibodies against cardiac troponin I were identified in PD-1 deficient mice and monoclonal antibodies reproduced the cardiac dysfunction observed in knockout animals (51). Non-classical immune cells can also regulate PD-1 signaling and control $\mathrm{T}$ cell activation. In a cytotoxic $\mathrm{T}$ lymphocyte model of myocarditis, PD-L1 was upregulated in endothelial cells by a mechanism dependent on IFN- $\gamma$ signaling. Abrogation of IFN- $\gamma$ signaling or PD-L1 genetic deletion transformed transient myocarditis to lethal disease, in association with leukocyte rich abscesses (52). Regulation of $\mathrm{CD}^{+} \mathrm{T}$ cell response was the major event controlling myocarditis, as demonstrated by increased cytotoxicity and activation profile of $\mathrm{CD} 8^{+} \mathrm{PD}-1$ knockout cells in myocarditis model. PD-L1 was also shown to prevent the development of heart autoimmunity in a model of graft versus host disease (53). In humans, PD-1 and PD-L1 proteins were detected in muscle tissue, preferentially in the heart (54) (www.proteinatlas.org).

It is therefore possible that inhibition of PD-1 signaling results in uncontrolled $\mathrm{T}$ cell activation and tolerance disruption within the heart (Figure 3). This hypothesis was confirmed in some patients receiving anti-PD1 antibodies for cancer treatment. In phase $1 \mathrm{~b}$ trial, combination of avelumab (anti-PD-L1) and axitinib (anti-VEGF) for renal-cell carcinoma resulted in grade 3 or worse treatment-related adverse effects, where the most frequent was hypertension (55).
Besides hypertension, which is possibly related to axitinib treatment, one patient died before data cutoff due to autoimmune myocarditis. In a phase II trial with pembrolizumab (anti-PD-1) for thymic epithelial tumor, $34 \%$ of patients displayed grade 3 or superior IRAE, where myocarditis developed in $9 \%$ and myasthenia gravis in $6 \%$ (56). Notably, heart IRAE were also present during a phase II study of Pembrolizumab for thymic carcinoma, in which six patients developed severe IRAE and two patients (5\%) present myocarditis and polymyositis after the second cycle of therapy (57). Patients immediately required high-dose steroid treatment and pacemaker placement, although one patient died of HF despite intervention. Corroborating other results, polyclonal $\mathrm{T}$ cell activation was found in the blood of myocarditis committed patients, with 26 TCR clones expanded in the blood and 7 in muscle biopsy (58). Tadokoro and coworkers reported a case of acute lymphocytic myocarditis in a melanoma patient that underwent 3 cycles of Nivolumab treatment. Left ventricular dysfunction and $\mathrm{CD} 8^{+} \mathrm{T}$ cell infiltration in myocardium were observed after 2 weeks since last treatment. While continuous dobutamine infusion did not improve cardiac function, high doses of prednisolone were sufficient to gradually improve the symptoms (59). Myocarditis was also observed in patient receiving Nivolumab for squamous cell lung carcinoma. Despite partial remission of lung tumor, drug induced myocarditis lead to immunotherapy stoppage and HF therapy with ACE-inhibitors, $\beta$-blockers, and steroids was initiated (60). Combination of tremelimumab (anti-CTLA-4) and durvalumab (anti-PD-L1) triggered myocarditis in an endometrial cancer patient, three weeks after last cycle. Immunotherapy stoppage and steroid administration over eight weeks were also necessary, which resulted in progression of metastatic disease (61).

Major heart adverse effects were also observed in a lung cancer patient treated with nivolumab in the presence of cytokine activated $\alpha \beta$ lymphocytes. Complete atrioventricular block and high levels of creatine kinasemyocardial b (CKMB) were observed after nine days of treatment, which could not be reversed despite high-dose steroid therapy (62). A similar case of fatal autoimmune myocarditis was observed in patient undergoing avelumab (anti-PD-L1) plus axitinib (tyrosine kinase inhibitor) for renal cell carcinoma. Fast onset of hypertension occurred after last axitinib treatment, and dose reduction was sufficient to control blood pressure levels. Despite the clinical absence of cardiovascular disease and only grade 1 
rise in creatinine kinase levels, patient collapsed and postmortem examination indicated massive infiltration of $\mathrm{CD}^{+}$ lymphocytes in myocardium, suggestive of autoimmune myocarditis (63). In some patients, concomitant autoimmune diseases were triggered after anti-PD-1 treatment. In that scenario, a patient receiving Nivolumab for lung cancer developed myocarditis and myasthenia gravis (MG). A fast IRAE was observed, after 3 weeks of last Nivolumab treatment. Immunohistochemical analysis revealed myocardium necrosis followed by $\mathrm{CD}^{+}$and $\mathrm{CD} 8^{+}$ $\mathrm{T}$ cell infiltration. Elevated serum levels of acetylcholine receptor antibody suggest the onset of MG (64). In a second case, patient receiving Nivolumab for pulmonary adenocarcinoma presented diabetic ketoacidosis, receiving the diagnosis of insulin-dependent diabetes mellitus, likely to anti-PD-1 treatment. It is worth noticing that the patient already presented risk factors for cardiovascular disease, such as hypertension and chronic obstructive pulmonary disease (COPD). Even though diabetes was controlled, lethargy and shortness of breath were reported. The final diagnosis indicated acute decompensated rightside HF and post-mortem analysis showed myocardial inflammation, necrosis, and $\mathrm{T}$ lymphocyte infiltration (65). Together, the use of immune checkpoint inhibitors targeting CTLA-4 and PD-1 signaling led to major cardiac adverse effects of autoimmune nature, which could not be reversed in some patients.

\section{TIGIT, TIM-3 and LAG-3}

Successful application of immune checkpoint inhibitors to tumors endowed with poor prognosis sparked the scientific community to extend its knowledge beyond CTLA-4 and PD-1 molecules. New inhibitory pathways that could potentially dampen $\mathrm{T}$ cell antitumor activity were discovered and currently blocking antibodies against those molecules are being investigated in first clinical trials (66).

Lymphocyte activation gene-3 (LAG-3) is expressed by $\mathrm{T}$ and NK cells following MHC class II ligation. Despite the exact mechanism remains largely unclear, LAG-3 activation results in negative regulatory effects over $\mathrm{T}$ cells (67). Analysis of tumor infiltrating lymphocytes revealed that LAG-3, along with PD-1, were highly expressed in those cells (67). LAG-3 signaling pathway is thought to induce $\mathrm{T}$ cell exhaustion and subsequent tumor growth and spread (68). Moreover, several tumor types express MHC II, the ligand for LAG-3, where they interaction promoted melanoma resistance to apoptosis (69). LAG-3 expression correlates with poor prognosis in head-neck carcinoma and inhibition of LAG-3 in mouse model favors $\mathrm{T}$ cell responses against tumor (70). Also, inhibition of LAG-3 alone or in combination of PD-1 resulted in improved $\mathrm{T}$ cell immunity against tumor cells followed by tumor rejection (71). As expected, two independent studies demonstrated that PD-1 and LAG-3 act synergistically to prevent autoimmunity $(72,73)$. While LAG-3 knockout did not induce autoimmunity per se, deficiency of LAG-3 and PD-1 resulted in lethal myocarditis (72). Recently a link between LAG-3 and coronary artery disease was established. Humans, which present the rs10846744 noncoding variant of lipoprotein scavenger receptor B1 (SCARB1), are more prone to develop atherosclerotic disease independently of common cardiovascular risks. Cells presenting the noncoding variant produced less amounts of LAG-3 under basal conditions and following BCR engagement. Plasma LAG-3 levels were found lower in hyperalphalipoproteinemia patients and significantly associate with HDL and IL-10 concentration in plasma (74). Therefore, LAG-3 may confer protective immune response in the heart, although this hypothesis was not formally addressed. Currently two phase I trials reported the effect of IMP321, a recombinant Ig which agonizes MHC II driven DCs activation (75). In a first study, CD8 $\mathrm{T}$ cell activation was increased along better progression-free survival. Minor adverse effects (grade 1) were reported. In a second study, combination of IMP321 with gemcitabine was used for pancreatic adenocarcinoma, but no treatment efficacy was observed after treatment (76). An initial efficacy of antiLAG3 (BMS-986016) in combination with Nivolumab was seen in melanoma patients. Grade 3 or superior adverse events were observed in $9 \%$ of patients, but descriptive results are still not available (77).

T-cell immunoglobulin-3 (TIM-3) is a molecule expressed on NK cells and macrophages which negatively regulates T cells activation (78). Mechanism wise, TIM3 has been shown to induce and expand myeloid derived suppressor cells (MDSCs) (79). Also, galectin-9, a TIM-3 ligand, induces intracellular calcium flux, aggregation, and death of Th1 cells in vitro. Moreover, in vivo administration results in selective loss of IFN- $\gamma$ producing cells and suppression of Th1 mediated autoimmunity (80). Exhausted T cells present high levels of TIM-3 and the presence of TIM- $3^{+}$cells is associated with poor prognosis in lung and follicular carcinoma $(81,82)$. For that reason, strategies that inhibit TIM-3 activation would greatly benefit $T$ cell response against tumor cells. In that scenario, Lee and 
coworkers showed that TIM-3 inhibition enhances efficacy of tumor vaccine in a mouse mode of lung carcinoma (83). Combined blockade of TIM-3 and MEK pathway increases $\mathrm{CD}^{+} \mathrm{T}$ cell response in a mouse model of melanoma (84). From studies investigating CTLA-4, PD-1, and LAG3 pathway, uncontrolled immune cell activation was anticipated. In a recent study, Zhang addressed the role of TIM-3 in CVB-3 mediated myocarditis. Exogenous administration of galectin-9 attenuated viral induced myocarditis by promoting the expansion of MDSCs. Production of Arg- 1 and IL-4 suppressed $\mathrm{CD}^{+}{ }^{+} \mathrm{T}$ cell proliferation in vitro (85). Using the CVB3 model of myocarditis, Frisancho-Kiss and coworkers described an increased inflammatory response in male BALB/C mice when compared to female mice. This phenotype was accompanied by increased TLR4 and IFN- $\gamma$ positive cells in the heart, whereas female hearts displayed a regulatory T cell phenotype, comprised of TIM-3, IL-4 and Treg. Importantly, inhibition of TIM-3 in male mice led to a significant increase in inflammation and TLR4 expression, while Tregs were significantly reduced (28). Currently antiTIM-3 mAbs (MBG453) are being under phase I-II clinical trial for advanced tumors. There are no results reported yet.

The T cell immunoreceptor Ig and ITIM domains (TIGIT) was recently classified as a member of immunoglobulin superfamily expressed on effector T cells, Tregs, NK cells, and in NKT population. TIGIT activation on $\mathrm{T}$ cells leads to proliferation inhibition, decrease in T-bet, GATA-3, IRF4, ROR- $\gamma$ t expression and ultimately reduced production of IFN- $\gamma$ (86). TIGIT can also control $\mathrm{T}$ cell activation in an indirect fashion, dependent on dendritic cells. Yu and coworkers demonstrated the biding of TIGIT to poliovirus receptor (PVR) in DCs, leading to enhanced IL-10 secretion and diminished IL-12p40 production (87). Besides those mechanisms, engagement of TIGIT in PVR and PVRL2 receptors present on human NK cells inhibits cytotoxicity through its ITIM domain (88). While TIGIT ligands are widely expressed on several epithelial cell types and on circulating monocytes, tumor cells also up-regulated PVR and PVRL2 as an immune escaping mechanism (89). Recent studies support this idea by showing the involvement of TIGIT in NK and $\mathrm{CD} 8^{+} \mathrm{T}$ cell exhaustion in melanoma and multiple myeloma models (44). Similarly to other immune checkpoint molecules, TIGIT deletion results in a hyperproliferative $\mathrm{T}$ cell response; antagonistic antibodies exacerbates experimental autoimmune encephalomyelitis $(90,91)$. Besides PVR, TIGIT can also recognize nectin molecules, resulting in inhibition of T and NK cell activation $(92,93)$. Nectins 1 , 2 , and 3 are expressed at different levels in heart tissue and Nectin-2 has been shown to maintain structure and function of intercalated disc and protect heart from pressure overload $(54,94)$. It is therefore possible that T- and NKcell surveillance in heart tissue mediated by TIGIT-Nectin interaction also contributes to a tolerogenic and protective type of immune response, although this has not been formally addressed yet. Meanwhile, a phase I trial is currently recruiting patients for safety and efficacy evaluation against anti-TIGIT mAb OMP-31M32. There are still no results available.

Based on previous cardiac related adverse events reported with classical ICI and data discussed about new checkpoint inhibitors, a careful analysis of cardiotoxicity should be included in clinical trials of next-generation ICI treatment.

\section{Concluding remarks}

Cardiac dysfunction in cancer patients during or after treatment is rapidly becoming a topic of high interest where an interdisciplinary approach is necessary to fulfil its needs. Only a deep understanding of the pathophysiology can lead to adequate treatment of cardiovascular side effects. While the introduction of immune checkpoint inhibitors improved the prognosis of several malignant cancers, it imposed new challenges for the scientific community. Major cardiac adverse effects were increasingly reported during and after ICI treatment. Mechanisms underlying such events, early detection of cardiotoxicity, and managing cardiac function post chemotherapy induced damage are still largely undetermined.

\section{Acknowledgements}

Funding: This work was supported by grants from the Bundesministerium für Bildung und Forschung (BMBF01 EO1004) and the IZKF (Interdisziplinäres Zentrum für Klinische Forschung).

\section{Footnote}

Conflicts of Interest: The authors have no conflicts of interest to declare.

\section{References}

1. Meijers WC, Maglione M, Bakker SJL, et al. The Failing Heart Stimulates Tumor Growth by Circulating Factors. Circulation 2018;138:678-91. 
2. Kostakou PM, Kouris NT, Kostopoulos VS, et al. Cardiooncology: a new and developing sector of research and therapy in the field of cardiology. Heart Fail Rev 2018. [Epub ahead of print].

3. Cardinale D, Colombo A, Lamantia G, et al. Anthracycline-Induced Cardiomyopathy. J Am Coll Cardiol 2010;55:213-20.

4. de Azambuja E, Bedard PL, Suter T, et al. Cardiac toxicity with anti-HER-2 therapies-what have we learned so far? Target Oncol 2009;4:77-88.

5. Zamorano JL, Lancellotti P, Rodriguez Muñoz D, et al. 2016 ESC Position Paper on cancer treatments and cardiovascular toxicity developed under the auspices of the ESC Committee for Practice Guidelines. Eur Heart J 2016;37:2768-801.

6. Lancellotti P, Suter TM, López-Fernández T, et al. Cardio-Oncology Services: rationale, organization, and implementation. Eur Heart J 2018. [Epub ahead of print]

7. Spranger S, Gajewski TF. Mechanism of Tumor CellIntrinsic Immune Evasion. Annu Rev Cancer Biol 2018;2:213-28.

8. Xing Y, Hogquist KA. T-Cell Tolerance: Central and Peripheral. Cold Spring Harb Perspect Biol 2012;4:a006957.

9. Leach DR, Krummel MF, Allison JP. Enhancement of antitumor immunity by CTLA-4 blockade. Science 1996;271:1734-6.

10. Dong H, Strome SE, Salomao DR, et al. Tumor-associated B7-H1 promotes T-cell apoptosis: A potential mechanism of immune evasion. Nat Med 2002;8:793-800.

11. Ribas A, Wolchok JD. Cancer immunotherapy using checkpoint blockade. Science 2018;359:1350-5.

12. Michot JM, Bigenwald C, Champiat S, et al. Immunerelated adverse events with immune checkpoint blockade: a comprehensive review. Eur J Cancer 2016;54:139-48.

13. Moslehi JJ, Salem J-E, Sosman JA, et al. Increased reporting of fatal immune checkpoint inhibitor-associated myocarditis. Lancet 2018;391:933.

14. Han X, Zhou Y, Liu W. Precision cardio-oncology: understanding the cardiotoxicity of cancer therapy. NPJ Precis Oncol 2017;1:31.

15. Chen L, Flies DB. Molecular mechanisms of T cell co-stimulation and co-inhibition. Nat Rev Immunol 2013;13:227-42.

16. Walker LSK, Sansom DM. The emerging role of CTLA4 as a cell-extrinsic regulator of $T$ cell responses. Nat Rev Immunol 2011;11:852-63.

17. Tivol EA, Borriello F, Schweitzer AN, et al. Loss of
CTLA-4 leads to massive lymphoproliferation and fatal multiorgan tissue destruction, revealing a critical negative regulatory role of CTLA-4. Immunity 1995;3:541-7.

18. Chambers CA, Sullivan TJ, Allison JP. Lymphoproliferation in CTLA-4-deficient mice is mediated by costimulation-dependent activation of CD4+ T cells. Immunity 1997;7:885-95.

19. Wing K, Onishi Y, Prieto-Martin P, et al. CTLA-4 Control over Foxp3+ Regulatory T Cell Function. Science 2008;322:271-5.

20. Kuehn HS, Ouyang W, Lo B, Deenick EK et al. Immune dysregulation in human subjects with heterozygous germline mutations in CTLA4. Science 2014;345:1623-7.

21. Crespo J, Sun H, Welling TH, et al. T cell anergy, exhaustion, senescence, and stemness in the tumor microenvironment. Curr Opin Immunol 2013;25:214-21.

22. Contardi E, Palmisano GL, Tazzari PL, et al. CTLA4 is constitutively expressed on tumor cells and can trigger apoptosis upon ligand interaction. Int $\mathrm{J}$ Cancer 2005;117:538-50.

23. Iwai $Y$, Ishida $M$, Tanaka $Y$, et al. Involvement of PD-L1 on tumor cells in the escape from host immune system and tumor immunotherapy by PD-L1 blockade. Proc Natl Acad Sci U S A 2002;99:12293-7.

24. Hodi FS, O’Day SJ, McDermott DF, et al. Improved Survival with Ipilimumab in Patients with Metastatic Melanoma. N Engl J Med 2010;363:711-23.

25. Varricchi G, Marone G, Mercurio V, et al. Immune Checkpoint Inhibitors and Cardiac Toxicity: An Emerging Issue. Curr Med Chem 2018;25:1327-39.

26. Lin H, Rathmell JC, Gray GS, et al. Cytotoxic T lymphocyte antigen 4 (CTLA4) blockade accelerates the acute rejection of cardiac allografts in CD28-deficient mice: CTLA4 can function independently of CD28. J Exp Med 1998;188:199-204.

27. Li S, Salgar SK, Thanikachalam M, et al. CTLA4-IgBased Conditioning Regimen to Induce Tolerance to Cardiac Allografts. J Surg Res 2006;136:238-46.

28. Frisancho-Kiss S, Davis SE, Nyland JF, et al. Cutting Edge: Cross-Regulation by TLR4 and T cell Ig Mucin-3 Determines Sex Differences in Inflammatory Heart Disease. J Immunol 2007;178:6710-4.

29. Han B, Jiang H, Liu Z, et al. CTLA4-Ig Relieves Inflammation in Murine Models of Coxsackievirus B3Induced Myocarditis. Can J Cardiol 2012;28:239-44.

30. Love VA, Grabie N, Duramad P. CTLA-4 Ablation and Interleukin-12 Driven Differentiation Synergistically Augment Cardiac Pathogenicity of Cytotoxic T 
Lymphocytes. Circ Res 2007;101:248-57.

31. Ruppert V, Meyer T, Struwe C, et al. Evidence for CTLA4 as a susceptibility gene for dilated cardiomyopathy. Eur J Hum Genet 2010;18:694-9.

32. Azevedo PM, Merriman TR, Topless RK, et al. Association study involving polymorphisms in IL-6, IL1RA, and CTLA4 genes and rheumatic heart disease in New Zealand population of Māori and Pacific ancestry. Cytokine 2016;85:201-6.

33. Dias FC, Medina T da S, Mendes-Junior CT, et al. Polymorphic Sites at the Immunoregulatory CTLA-4 Gene Are Associated with Chronic Chagas Disease and Its Clinical Manifestations. PLoS One 2013;8:e78367.

34. Johnson DB, Balko JM, Compton ML, et al. Fulminant Myocarditis with Combination Immune Checkpoint Blockade. N Engl J Med 2016;375:1749-55.

35. Geisler BP, Raad RA, Esaian D, et al. Apical ballooning and cardiomyopathy in a melanoma patient treated with ipilimumab: a case of takotsubo-like syndrome. J Immunother Cancer 2015;3:4.

36. Samara Y, Yu CL, Dasanu CA. Acute autoimmune myocarditis and hepatitis due to ipilimumab monotherapy for malignant melanoma. J Oncol Pharm Pract 2018;107815521875586. [Epub ahead of print].

37. Jain V, Mohebtash M, Rodrigo ME, et al. Autoimmune Myocarditis Caused by Immune Checkpoint Inhibitors Treated With Antithymocyte Globulin. J Immunother 2018;41:332-5.

38. Reuben A, Petaccia de Macedo M, McQuade J, et al. Comparative immunologic characterization of autoimmune giant cell myocarditis with ipilimumab. OncoImmunology 2017;6:e1361097.

39. Mahmood SS, Fradley MG, Cohen JV, et al. Myocarditis in Patients Treated With Immune Checkpoint Inhibitors. J Am Coll Cardiol 2018;71:1755-64.

40. Yun S, Vincelette ND, Mansour I, et al. Late Onset Ipilimumab-Induced Pericarditis and Pericardial Effusion: A Rare but Life Threatening Complication. Case Rep Oncol Med 2015;2015:794842.

41. Norwood TG, Westbrook BC, Johnson DB, et al. Smoldering myocarditis following immune checkpoint blockade. J Immunother Cancer 2017;5:91.

42. Sharpe AH, Pauken KE. The diverse functions of the PD1 inhibitory pathway. Nat Rev Immunol 2018;18:153-67.

43. Freeman GJ, Long AJ, Iwai Y, et al. Engagement of the PD-1 immunoinhibitory receptor by a novel B7 family member leads to negative regulation of lymphocyte activation. J Exp Med 2000;192:1027-34.
44. Chauvin J-M, Pagliano O, Fourcade J, et al. TIGIT and PD-1 impair tumor antigen-specific CD8+ T cells in melanoma patients. J Clin Invest 2015;125:2046-58.

45. Topalian SL, Hodi FS, Brahmer JR, et al. Safety, Activity, and Immune Correlates of Anti-PD-1 Antibody in Cancer. N Engl J Med 2012;366:2443-54.

46. Topalian SL, Sznol M, McDermott DF, et al. Survival, Durable Tumor Remission, and Long-Term Safety in Patients With Advanced Melanoma Receiving Nivolumab. J Clin Oncol 2014;32:1020-30.

47. Heinzerling L, Ott PA, Hodi FS, et al. Cardiotoxicity associated with CTLA4 and PD1 blocking immunotherapy. J Immunother Cancer 2016;4:50.

48. Nishimura H., Nose M, Hiai H, et al. Development of lupus-like autoimmune diseases by disruption of the PD-1 gene encoding an ITIM motif-carrying immunoreceptor. Immunity 1999;11:141-51.

49. Wang J, Yoshida T, Nakaki F, et al. Establishment of NOD-Pdcd1-/- mice as an efficient animal model of type I diabetes. Proc Natl Acad Sci U S A 2005;102:11823-8.

50. Nishimura H, Okasaki T, Tanaka Y, et al. Autoimmune Dilated Cardiomyopathy in PD-1 Receptor-Deficient Mice. Science 2001;291:319-22.

51. Okazaki T, Tanaka Y, Nishio R, et al. Autoantibodies against cardiac troponin I are responsible for dilated cardiomyopathy in PD-1-deficient mice. Nat Med 2003;9:1477-83.

52. Grabie N, Gotsman I, DaCosta R, et al. Endothelial Programmed Death-1 Ligand 1 (PD-L1) Regulates CD8+ T-Cell-Mediated Injury in the Heart. Circulation 2007;116:2062-71.

53. Juchem KW, Sacirbegovic F, Zhang C, et al. PD-L1 Prevents the Development of Autoimmune Heart Disease in Graft-versus-Host Disease. J Immunol 2018;200:834-46.

54. Uhlén M, Fagerberg L, Hallstrom BM, et al. Tissue-based map of the human proteome. Science 2015;347:1260419.

55. Choueiri TK, Larkin J, Oya M, et al. Preliminary results for avelumab plus axitinib as first-line therapy in patients with advanced clear-cell renal-cell carcinoma (JAVELIN Renal 100): an open-label, dose-finding and doseexpansion, phase 1b trial. Lancet Oncol 2018;19:451-60.

56. Cho J, Kim HS, Ku BM, et al. Pembrolizumab for Patients With Refractory or Relapsed Thymic Epithelial Tumor: An Open-Label Phase II Trial. J Clin Oncol 2018. [Epub ahead of print].

57. Giaccone G, Kim C, Thompson J, et al. Pembrolizumab in patients with thymic carcinoma: a single-arm, singlecentre, phase 2 study. Lancet Oncol 2018;19:347-55. 
58. Varricchi G, Galdiero MR, Marone G, et al. Cardiotoxicity of immune checkpoint inhibitors. ESMO Open 2017;2:e000247.

59. Tadokoro T, Keshino E, Makiyama A, et al. Acute Lymphocytic Myocarditis With Anti-PD-1 Antibody Nivolumab. Circ Heart Fail 2016;9:e003514.

60. Semper H, Muehlberg F, Schulz-Menger J, et al. Druginduced myocarditis after nivolumab treatment in a patient with PDL1- negative squamous cell carcinoma of the lung. Lung Cancer 2016;99:117-9.

61. Mahmood SS, Chen CL, Shapnik N, et al. Myocarditis with tremelimumab plus durvalumab combination therapy for endometrial cancer: A case report. Gynecol Oncol Rep 2018;25:74-7.

62. Kimura H, Tounai Y, Nagato K, et al. A Case of Heart Fail after Treatment with Anti-PD-1 Antibody Followed by Adoptive Transfer of Cytokine-Activated Killer Cells in a Recurrent Lung Cancer Patient. J Thorac Oncol 2017;12:e128-30.

63. Berner AM, Sharma A, Agarwal S, et al. Fatal autoimmune myocarditis with anti-PD-L1 and tyrosine kinase inhibitor therapy for renal cell cancer. Eur J Cancer 2018;101:287-90.

64. Fukasawa Y, Sasaki K, Natsume M, et al. NivolumabInduced Myocarditis Concomitant with Myasthenia Gravis. Case Rep Oncol 2017;10:809-12.

65. Matson DR, Accola MA, Rehrauer WM, et al. Fatal Myocarditis Following Treatment with the PD-1 Inhibitor Nivolumab. J Forensic Sci 2018;63:954-7.

66. Marin-Acevedo JA, Dholaria B, Soyano AE, et al. Next generation of immune checkpoint therapy in cancer: new developments and challenges. J Hematol Oncol 2018;11:39.

67. Andrews LP, Marciscano AE, Drake CG, et al. LAG3 (CD223) as a cancer immunotherapy target. Immunol Rev 2017;276:80-96.

68. Demeure CE, Wolfers J, Martin-Garcia N, et al. T Lymphocytes infiltrating various tumour types express the MHC class II ligand lymphocyte activation gene-3 (LAG3): role of LAG-3/MHC class II interactions in cell-cell contacts. Eur J Cancer 2001;37:1709-18.

69. Hemon P, Jean-Louis F, Ramgolam K, et al. MHC Class II Engagement by Its Ligand LAG-3 (CD223) Contributes to Melanoma Resistance to Apoptosis. J Immunol 2011;186:5173-83.

70. Deng WW, Mao L, Yu GT, et al. LAG-3 confers poor prognosis and its blockade reshapes antitumor response in head and neck squamous cell carcinoma. OncoImmunology 2016;5:e1239005.
71. Goding SR, Wilson KA, Xie Y, et al. Restoring Immune Function of Tumor-Specific CD4+ T Cells during Recurrence of Melanoma. J Immunol 2013;190:4899-909.

72. Okazaki T, Okazaki I, Wang J, et al. PD-1 and LAG3 inhibitory co-receptors act synergistically to prevent autoimmunity in mice. J Exp Med 2011;208:395-407.

73. Woo SR, Turnis ME, Goldberg MV, et al. Immune Inhibitory Molecules LAG-3 and PD-1 Synergistically Regulate T-cell Function to Promote Tumoral Immune Escape. Cancer Res 2012;72:917-27.

74. Golden D, Kolmakova A, Sura S, et al. Lymphocyte activation gene 3 and coronary artery disease. JCI Insight 2016;1:e88628.

75. Brignone C, Escudier B, Grygar C, et al. A Phase I Pharmacokinetic and Biological Correlative Study of IMP321, a Novel MHC Class II Agonist, in Patients with Advanced Renal Cell Carcinoma. Clin Cancer Res 2009;15:6225-31.

76. Wang-Gillam A, Plambeck-Suess S, Goedegebuure P et al. A phase I study of IMP321 and gemcitabine as the front-line therapy in patients with advanced pancreatic adenocarcinoma. Invest New Drugs 2013;31:707-13.

77. Ascierto PA, Melero I, Bhatia S, et al. Initial efficacy of anti-lymphocyte activation gene-3 (anti-LAG-3; BMS986016) in combination with nivolumab (nivo) in pts with melanoma (MEL) previously treated with anti-PD-1/PDL1 therapy. J Clin Oncol 2017;35:9520.

78. Anderson AC, Joller N, Kuchroo VK. Lag-3, Tim-3, and TIGIT: Co-inhibitory Receptors with Specialized Functions in Immune Regulation. Immunity 2016;44:989-1004.

79. Dardalhon V, Anderson AC, Karman J, et al. Tim-3/ Galectin-9 Pathway: Regulation of Th1 Immunity through Promotion of CD11b+Ly-6G+ Myeloid Cells. J Immunol 2010;185:1383-92.

80. Zhu C, Anderson AC, Schubart A, et al. The Tim-3 ligand galectin-9 negatively regulates $\mathrm{T}$ helper type 1 immunity. Nat Immunol 2005;6:1245-52.

81. Zhang Y, Cai P, Liang T, et al. TIM-3 is a potential prognostic marker for patients with solid tumors: A systematic review and meta-analysis. Oncotarget 2017;8:31705-13.

82. Su H, Xie H, Dai C, et al. Characterization of TIM3 expression and its prognostic value in patients with surgically resected lung adenocarcinoma. Lung Cancer 2018;121:18-24.

83. Lee MJ, Woo M-Y, Heo YM, et al. The inhibition of the T-cell immunoglobulin and mucin domain 3 (Tim3) pathway enhances the efficacy of tumor vaccine. Biochem 
Biophys Res Commun 2010;402:88-93.

84. Liu Y, Cai P, Wang N et al. Combined blockade of Tim-3 and MEK inhibitor enhances the efficacy against melanoma. Biochem Biophys Res Commun 2017;484:378-84.

85. Zhang Y, Zhang M, Li X, et al. Expansion of CD11b + Ly$6 \mathrm{C}+$ myeloid-derived suppressor cells (MDSCs) driven by galectin-9 attenuates CVB3-induced myocarditis. Mol Immunol 2017;83:62-71.

86. Lozano E, Dominguez-Villar M, Kuchroo V, Hafler DA. The TIGIT/CD226 Axis Regulates Human T Cell Function. J Immunol 2012;188:3869-75.

87. Yu X, Harden K, Gonzalez LC, et al. The surface protein TIGIT suppresses $\mathrm{T}$ cell activation by promoting the generation of mature immunoregulatory dendritic cells. Nat Immunol 2009;10:48-57.

88. Stanietsky N, Simic H, Arapovic J, et al. The interaction of TIGIT with PVR and PVRL2 inhibits human NK cell cytotoxicity. Proc Natl Acad Sci U S A 2009;106:17858-63.

89. Masson D, Jarry A, Baury B, et al. Overexpression of the CD155 gene in human colorectal carcinoma. Gut

Cite this article as: Delgobo $M$, Frantz S. Heart failure in cancer: role of checkpoint inhibitors. J Thorac Dis 2018;10(Suppl 35):S4323-S4334. doi: 10.21037/jtd.2018.10.07
2001;49:236-40.

90. Joller N, Hafler JP, Brynedal B, et al. Cutting Edge: TIGIT Has T Cell-Intrinsic Inhibitory Functions. J Immunol 2011;186:1338-42.

91. Dixon KO, Schorer M, Nevin J, et al. Functional Anti-TIGIT Antibodies Regulate Development of Autoimmunity and Antitumor Immunity. J Immunol 2018;200:3000-7.

92. Stanietsky N, Rovis TL, Glasner A, et al. Mouse TIGIT inhibits NK-cell cytotoxicity upon interaction with PVR. Eur J Immunol 2013;43:2138-50.

93. Deuss FA, Gully BS, Rossjohn J, et al. Recognition of nectin-2 by the natural killer cell receptor $\mathrm{T}$ cell immunoglobulin and ITIM domain (TIGIT). J Biol Chem 2017;292:11413-22.

94. Satomi-Kobayashi S, Ueyama T, Mueller S, et al. Deficiency of Nectin-2 Leads to Cardiac Fibrosis and Dysfunction Under Chronic Pressure Overload. Hypertension 2009;54:825-31. 